\title{
Development and Validation of a Dementia Risk Score in the UK Biobank and Whitehall II Cohorts
}

\author{
Melis Anatürk $\pm^{\mathrm{a}, \mathrm{b}}$, Raihaan Patel $\pm^{\mathrm{b}, \mathrm{c}^{*}}$, Klaus P. Ebmeier ${ }^{\mathrm{b}}$, Georgios Georgiopoulos ${ }^{\mathrm{d}}$, Danielle \\ Newby $^{\mathrm{b}}$, Anya Topiwala ${ }^{\mathrm{b}, \mathrm{e}}$, Ann-Marie G. de Lange ${ }^{\mathrm{b}, \mathrm{f}, \mathrm{g}}$, James H. Cole ${ }^{\mathrm{a}, \mathrm{h}}$, Michelle G. Jansen', \\ Archana Singh-Manoux ${ }^{\mathrm{j}, \mathrm{k}}$, Mika Kivimäkik, Sana Suri ${ }^{\mathrm{b}, \mathrm{c}}$ \\ ${ }^{a}$ Centre for Medical Image Computing, Department of Computer Science, University College London, \\ London, UK \\ ${ }^{\mathrm{b}}$ Department of Psychiatry, University of Oxford, Oxford, UK \\ ${ }^{\circ}$ Oxford Centre for Human Brain Activity, Wellcome Centre for Integrative Neuroimaging, University of \\ Oxford, Oxford, UK \\ d School of Biomedical Engineering and Imaging Sciences, King's College London, London UK

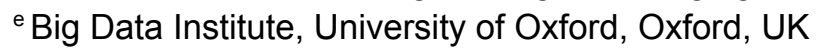 \\ ${ }^{\mathrm{f}}$ Department of Clinical Neurosciences, LREN, CHUV, University of Lausanne, Lausanne, Switzerland \\ g Department of Psychology, University of Oslo, Oslo, Norway \\ ${ }^{\mathrm{h}}$ Dementia Research Centre, Institute of Neurology, University College London, London, UK \\ 'Donders Centre for Cognition, Donders Institute for Brain, Cognition and Behaviour, Radboud University, \\ Nijmegen, the Netherlands \\ ${ }^{\mathrm{j}}$ Epidemiology of Ageing and Neurodegenerative diseases, Université de Paris, INSERM U1153, Paris, \\ France \\ ${ }^{\mathrm{k}}$ Department of Epidemiology and Public Health, University College London, London, UK \\ \pm Joint first author
}

${ }^{*}$ Corresponding Author:

Dr Raihaan Patel

Oxford Centre for Human Brain Activity

Wellcome Centre for Integrative Neuroimaging

Department of Psychiatry

University of Oxford

Oxford, UK

Email: mohammed.patel@psych.ox.ac.uk 


\section{Abstract}

Background: Current dementia risk scores have had limited success in consistently identifying at-risk individuals across different ages and geographical locations.

Objective: We aimed to develop and validate a novel dementia risk score for a midlife UK population, using two cohorts: the UK Biobank and UK Whitehall II Study.

Methods: We divided the UK Biobank cohort into a training $(n=176,611,80 \%)$ and test sample $(n=44,151,20 \%)$ and used the Whitehall II cohort $(n=3,028)$ for external validation. We used logistic LASSO regression to select the strongest predictors of incident dementia from thirty candidate predictors and then developed the risk score using logistic regression.

Findings: Our risk score, termed the UK Biobank Dementia Risk Score (UKBDRS), consisted of age, education, parental history of dementia, material deprivation, a history of diabetes, stroke, depression, hypertension, and high cholesterol. The score had a strong discrimination accuracy in the UK Biobank test sample $(\mathrm{AUC}[95 \% \mathrm{Cl}]=0.77[0.75,0.78])$ and in the Whitehall cohort $(0.75[0.7,0.79])$. The UKBDRS also significantly outperformed three other widely used dementia risk scores originally developed in cohorts in Australia (the Australian National University Alzheimer's Disease Risk Index), Finland (the Cardiovascular Risk Factors, Aging, and Dementia score), and the UK (Dementia Risk Score).

Clinical Implications: Our risk score represents an easy-to-use tool to identify individuals at risk for dementia in the UK. Further research is required to determine the validity of this score in other populations. 
What is already known on this topic

- Current dementia risk scores have limited generalizability

What this study adds

- We develop the UKBDRS - a dementia risk score based on large UK-based cohorts which outperforms three commonly used scores

How this study might affect research, practice, or policy

- The UKBDRS represents a scalable tool for dementia risk stratification in the UK

- Our results suggest caution when applying risk scores to external populations 


\section{BACKGROUND}

An estimated 50 million individuals are currently living with dementia worldwide[1]. With the number of dementia cases projected to triple by 2050 , prevention is a crucial avenue for addressing this public health challenge[1]. Up to $40 \%$ of dementia cases may be prevented by targeting 12 key risk factors, including low education levels, smoking, hypertension, obesity, diabetes and excessive alcohol intake[2]. Prognostic models of dementia risk which incorporate these factors may help identify high-risk individuals while they are still in the prodromal phases and direct them towards interventions to delay or prevent dementia[3].

Several prognostic models have been developed to predict individual-level dementia risk. These models vary in the factors used to predict risk, and have previously included various combinations of sociodemographic, cognitive, imaging, biomedical and genetic variables[4]. However, while the availability and use of dementia risk scores is increasing, there are many limitations which still need to be addressed[5].

For instance, risk scores are often developed in a single population without external validation. While increased attention to this issue has been paid in recent years[6, 7], problems with external validation still persist. For instance, a 2019 systematic review of 61 available dementia risk scores found that only 8 had been externally validated[3]. Moreover, those that had been validated often had poor and inconsistent performance in external samples. An external validation of four commonly used prediction models in a Dutch population found that only one model performed strongly (c-statistic $>0.8$ ) and all models were poorly calibrated with underestimation of low risk and overestimation of higher risk[6]. A recent large-scale validation

study assessed performance of 17 risk models in an Icelandic population and found that only models which included comprehensive cognitive testing achieved moderate prediction accuracy (c-statistic>0.75), while all other models performed poorly (c-statistic<0.75)[8]. 
Several reasons may underlie the poor out-of-sample performance of dementia risk scores. First, the factors associated with dementia, as well the importance of each factor, may vary across different geographical locations. Most development cohorts are typically from North America, with few others coming from across Europe[3, 8]. To our knowledge, there is only one dementia risk model that has been developed for the UK population, and little is known about how other externally-developed dementia risk models may perform in the UK population[9]. Second, the risk factors associated with dementia may vary across the lifespan. Interestingly, Hou et al. noted that only four risk scores have been developed for use in mid-life compared to 39 developed for use in elderly populations[3]. Thus, many of the available models may not be applicable to adults in mid-life who stand to benefit the most from early interventions. Third, model overfitting could underlie poor out-of-sample performance, particularly as until recently, the standard approach for developing risk scores involved conducting numerous univariate models to identify the best predictors of dementia from a long list of candidate predictors. Many dementia risk scores may integrate invasive (cerebrospinal fluid), time-intensive (cognitive batteries), or expensive (magnetic resonance imaging) markers. While such scores may offer modest improvements of prediction accuracy, they are more appropriate for specialised clinical settings rather than routine primary care or for population screening where these measurements are not available at scale[5].

\section{OBJECTIVES}

In this study, we address these limitations by using data from the UK Biobank (UKB) study to develop a novel dementia risk score specific to a mid-life UK population, which we refer to as the UK Biobank Dementia Risk Score (UKBDRS). We externally validate our score in the Whitehall II Study, another UK-based cohort with mid-life assessments. We focus on easily accessible sociodemographic and clinical data to enable the wider use of the score for routine care or large-scale screening for preventative trials. To combat the challenge of overfitting, we 
employ regularized regression methods to select variables used in the UKBDRS. We also compare the performance of our score to three previously published dementia risk scores developed in cohorts in Australia (the Australian National University Alzheimer's Disease Risk Index, ANU-ADRI[10]), Finland (the Cardiovascular Risk Factors, Aging, and Dementia score, CAIDE[11]), and the UK (Dementia Risk Score, DRS[9]). We chose these other scores as they are widely used in research studies worldwide, have previously been validated in at least one external cohort[3, 6], are composed of easily accessible risk factors, and therefore offer a reasonable comparative framework against which to test our novel score.

\section{METHODS}

\section{Sample Selection}

The UKBDRS was developed and validated internally in the UK Biobank Study (UKB) and tested externally in the Whitehall II Study cohort (WHII). The UKB[12] is a longitudinal cohort study of over 500,000 individuals (age range: 40-69). The WHII cohort[13] includes 10,308 British civil servants recruited in 1985 (age range: 35-55) who have since received comprehensive clinical examinations approximately every five years across 12 study waves. In both samples, we excluded individuals younger than 50 years old in order to focus our analysis on those with increased likelihood of developing dementia within the study follow-up period and reduce the likelihood of including those with monogenic risk of developing dementia. We also excluded individuals with missing data on any of the candidate risk factors described. For detailed sample selection, inclusion/exclusion criteria and comparison of included and excluded participants, please see SI Fig. 1, SI Table 2 and the SI Methods. The UKB sample was split into a training set ( $80 \%$ of sample) and test set $(20 \%)$, stratified for proportion of incident cases. APOE genotype is a strong, but less readily available, risk factor. Thus, we performed a second analysis to compute a version of the UKBDRS including APOE genotype. 
The UKB study received ethical approval from the Northwest Multi-Centre Research Ethics Committee (MREC) and the WHII study obtained ethical approval from the University College London Medical School Committee on the Ethics of Human Research. All participants provided their informed written consent.

\section{Dementia Ascertainment}

In the UKB sample, all-cause dementia status was determined based on complementary sources of information as done in several papers based on this cohort[14, 15]. An individual was classified as having dementia if they had either (1) self-reported a diagnosis at baseline (excluded from analyses), (2) received a primary or secondary diagnosis of dementia (primary care/ hospital records), (3) were prescribed dementia-related medications (e.g., rivastigmine) by their general practitioner (GP), or (4) if their primary or secondary cause of death was dementia-related. UKB Field IDs used for diagnosis and the list of ICD-9 and ICD-10 codes presented in the SI Table 3. In the WHII sample, dementia diagnosis was determined through self-report and hospital inpatient records[16, 17] (SI Methods, SI Table 3).

\section{Identifying Candidate Predictors}

We compiled a list of 28 risk and protective factors associated with dementia, including the 12 modifiable factors identified by the Lancet Commission[2]. Predictors were selected for inclusion if (1) they had been consistently associated with dementia, (2) if information about these was available in UKB, and (3) they could be easily obtained within a primary care setting. The full list of predictors is available in SI Table 1, with detailed descriptions including UKB field codes in SI Methods and SI Table 4.

\section{Construction of Existing Risk Scores}

The predictive ability of the UKBDRS was compared with that of three existing risk scores: The DRS[9], ANU-ADRI[10], and CAIDE[11]. We computed the three risk scores in the 
UKB and WHII cohorts using the formulae reported in the respective original papers (SI Methods).

\section{Statistical Analyses: Development of the UKBDRS}

All continuous variables were standardised and outliers (i.e., individuals with values $<$ Q1 - $3 \times$ interquartile range $(I Q R)$ or values $>Q 3+3 \times I Q R)$ were excluded $(1.8 \%$ of dataset $)$. The first stage ('variable selection') used only the training set and involved submitting the 28 candidate predictors to a least absolute shrinkage and selection operator (LASSO) logistic regression (SI Methods), with dementia diagnosis as outcome to identify a parsimonious model[18]. LASSO selected variables (SI Table 5) were then used as predictors in a standard logistic regression model with dementia status as the outcome. The linear predictor (intercept + beta coefficients) of each model was used to compute the predicted probability of developing dementia. Two variants of the UKBDRS were derived: a score without APOE (UKBDRS) and a score with APOE (UKBDRS-APOE). Our primary model is the UKBDRS, as genetic information may not be widely available.

\section{Statistical Analyses: Evaluation of the UKBDRS}

The performance of the UKBDRS was compared to the DRS, CAIDE, and ANU-ADRI in the UKB train, test and WHII datasets. All models were additionally compared to a baseline model consisting of chronological age only, to examine the added predictive value of additional factors. The model discrimination was evaluated using the area under the curve (AUC). DeLong's test[19] was used for pairwise comparisons of the AUCs of the UKBDRS with the other risk models. P-values were corrected for multiple comparisons (false discovery rate (FDR) p-value $\leq 0.05$ considered significant).

We used risk-calibration to assess the agreement between the observed proportion of dementia cases and predicted probabilities of developing dementia as calculated from the risk score (SI Methods). This study is reported in accordance with the Transparent Reporting of a 
Multivariable Prediction Model for Individual Prognosis or Diagnosis (TRIPOD) statement. Data analysis was conducted in R (3.6.3) and Python (3.6), using FSL's funpack and using the following R packages: CARET[20], glmnet[21], pROC[22], and rms[23].

\section{Sensitivity Analyses}

We also evaluated performance of each score only in the age ranges for which the external scores were originally developed. To do this we truncated the UKB Test and WHII cohorts to match the age range of original development and validation cohorts and computed the AUC. We also assessed how performance of the score was affected by time to dementia diagnosis (i.e., receiving a diagnosis 1-5 years, 5-10 years, or 10-14 years since baseline).

\section{Data and Code Availability}

The data used in this study are available from the UK Biobank (https://www.ukbiobank.ac.uk/enable-your-research/apply-for-access) and DPUK portal (https://portal.dementiasplatform.uk/Apply). As restrictions apply to the availability of these data, which were used under licence for the current study, the authors cannot publicly share this data. The use of data from the UK Biobank was approved by the UK Biobank Access Committee (Project No. 47279), while the use of data from the WHIl study was approved by the DPUK Access Committee (Project No. 0346). Code is available at https://github.com/MelisAnaturk/dementia_risk_score.

\section{FINDINGS}

\section{Sample Characteristics}

220,762 (mean age=59.97, SD=5.43) and 3,028 (median age=57, IQR=10) individuals were in the analysis samples for UKB and WHII, respectively. The sample characteristics are in SI Table 1, and participant selection is in SI Fig. 1. $3816(1.7 \%)$, and $96(3.2 \%)$ participants developed dementia in the UKB and WHII cohorts respectively. The maximum years to 
diagnosis in UKB was 14 years, which was used as the time window for dementia prediction for our risk score. There were significant differences in age, sex, and education between included and excluded participants in UKB and WHII (SI Table 2). The UKBDRS-APOE was computed on subsets with complete APOE information ( $N=157,090$ for UKB and $N=2,341$ for WHII).

\section{Selection of predictors for the UKBDRS}

The LASSO regression identified nine variables as predictive of incident dementia: age, education, history of diabetes, history/current depression, history of stroke, parental history of dementia, being in the most deprived Townsend Deprivation group, hypertension, and high cholesterol (SI Table 5). Though sex was not selected as a predictive factor by LASSO regression, based on evidence suggesting a predictive role of sex in dementia we tested performance of our score by adding sex to our logistic regression model, as well as when fitting sex-specific beta coefficients. Neither resulted in improved performance, and thus sex is left out of our final model. The beta coefficients for the final models are provided in Table 1. 
Table 1: Results of the logistic regressions with two variants of the UKBDRS. For each predictor, the beta coefficient is presented along with it's $95 \%$ confidence interval $(\mathrm{Cl})$, odds ratio (OR), and p-value.

\begin{tabular}{|c|c|c|c|c|c|}
\hline Predictor & $\beta$ & $\begin{array}{r}9 \\
\text { Lower }\end{array}$ & I Upper & OR $[95 \% \mathrm{Cl}]$ & $p$ \\
\hline \multicolumn{6}{|c|}{ UKBDRS } \\
\hline (Intercept) & -15.09 & -15.7 & -14.48 & & $2.1 \times 10^{-296}$ \\
\hline Age (Years) & 0.18 & 0.171 & 0.189 & $1.2[1.19,1.21]$ & $2.1 \times 10^{-296}$ \\
\hline $\begin{array}{c}\text { Parental History } \\
\text { (Yes) }\end{array}$ & 0.412 & 0.325 & 0.498 & $1.51[1.38,1.65]$ & $1.63 \times 10^{-20}$ \\
\hline $\begin{array}{l}\text { Education } \\
\text { (Years) }\end{array}$ & -0.044 & -0.056 & -0.032 & $0.96[0.95,0.97]$ & $5.45 \times 10^{-12}$ \\
\hline $\begin{array}{c}\text { Townsend } \\
\text { Deprivation } \\
\text { (most deprived) }\end{array}$ & 0.245 & 0.159 & 0.33 & $1.28[1.17,1.39]$ & $2.45 \times 10^{-8}$ \\
\hline Diabetes (Yes) & 0.549 & 0.431 & 0.664 & $1.73[1.54,1.94]$ & $5.66 \times 10^{-20}$ \\
\hline Depression (Yes) & 0.532 & 0.439 & 0.623 & $1.7[1.55,1.86]$ & $2.11 \times 10^{-29}$ \\
\hline Stroke (Yes) & 0.682 & 0.523 & 0.837 & $1.98[1.69,2.31]$ & $2.81 \times 10^{-17}$ \\
\hline $\begin{array}{l}\text { Hypertensive } \\
\text { (yes) }\end{array}$ & 0.178 & 0.097 & 0.259 & $1.2[1.1,1.3]$ & $1.83 \times 10^{-5}$ \\
\hline $\begin{array}{c}\text { High Cholesterol } \\
\text { (yes) }\end{array}$ & 0.123 & 0.035 & 0.211 & $1.13[1.04,1.24]$ & $6.18 \times 10^{-3}$ \\
\hline \multicolumn{6}{|c|}{ UKBDRS-APOE } \\
\hline (Intercept) & -16.15 & -16.887 & -15.453 & & 0 \\
\hline Age (Years) & 0.189 & 0.179 & 0.2 & $1.21[1.2,1.22]$ & $1.56 \times 10^{-268}$ \\
\hline $\begin{array}{c}\text { Parental History } \\
\text { (Yes) }\end{array}$ & 0.285 & 0.185 & 0.385 & $1.33[1.2,1.47]$ & $3.1 \times 10^{-8}$ \\
\hline $\begin{array}{l}\text { Education } \\
\text { (Years) }\end{array}$ & -0.039 & -0.053 & -0.025 & $0.96[0.95,0.98]$ & $1.14 \times 10^{-7}$ \\
\hline $\begin{array}{c}\text { Townsend } \\
\text { Deprivation } \\
\text { (most deprived) }\end{array}$ & 0.277 & 0.177 & 0.376 & $1.32[1.19,1.46]$ & $6.5 \times 10^{-8}$ \\
\hline Diabetes (Yes) & 0.548 & 0.407 & 0.687 & $1.71[1.5,1.9]$ & $3.35 \times 10^{-14}$ \\
\hline
\end{tabular}




\begin{tabular}{|c|c|c|c|c|c|}
\hline Depression (Yes) & 0.537 & 0.428 & 0.643 & $1.71[1.53,1.9]$ & $4.53 \times 10^{-22}$ \\
\hline Stroke (Yes) & 0.686 & 0.494 & 0.87 & $1.99[1.64,2.39]$ & $1.43 \times 10^{-12}$ \\
\hline $\begin{array}{c}\text { Hypertensive } \\
\text { (yes) }\end{array}$ & 0.207 & 0.112 & 0.301 & $1.23[1.23,1.35]$ & $1.99 \times 10^{-5}$ \\
\hline $\begin{array}{c}\text { High Cholesterol } \\
\text { (yes) }\end{array}$ & 0.04 & -0.062 & 0.142 & $1.04[0.94,1.15]$ & $4.4 \times 10^{-1}$ \\
\hline $\begin{array}{c}\text { APOE4 } \\
\text { genotype (yes) }\end{array}$ & 1.16 & 1.075 & 1.247 & $3.19[2.93,3.48]$ & $3.48 \times 10^{-155}$ \\
\hline
\end{tabular}

\section{Discrimination and Calibration in the UKB and WHII test sets}

In the UKB test sample, the AUC of the UKBDRS was $0.77(95 \% \mathrm{CI}=[0.75,0.78])$, while the UKBDRS-APOE achieved an AUC of $0.80(95 \% \mathrm{Cl}=[0.79,0.82])$ in the subset of UKB individuals with available genotype data. In the WHII sample, AUC of the UKBDRS was 0.75 (95 $\% \mathrm{Cl}=[0.7,0.79])$, and AUC of the UKBDRS-APOE was $0.79(95 \% \mathrm{Cl}=[0.75,0.84])($ Table 2$)$. Sensitivity analyses showed that the UKBDRS performed better in predicting dementia 10-14 years later $(A U C=0.79[0.76,0.81])$ compared to prediction of dementia $1-5$ years $(A U C=0.75$ $[0.71,0.8])$ or $5-10$ years later $(\mathrm{AUC}=0.75[0.73,0.78])(\mathrm{SI}$ Table 6$)$

In both the UKB test sample and the external WHII validation, the UKBDRS had significantly higher AUCs compared to the age-only model, the DRS, CAIDE, and the ANU-ADRI $\left(p_{\text {corr }}<0.05\right.$, Table 3 ). Figure 1 plots the ROC curves for all models. In the UKB training and test samples, the UKBDRS consistently outperformed external risk models even when we restricted the analysis to the age range for which each external score was originally developed. In the WH sample, the UKBDRS model outperformed the CAIDE but performed similarly to the DRS and ANU-ADRI in individuals over 60 (SI Table 7). SI Table 8 presents sensitivity, specificity, negative predictive (NPV) and positive predictive values (PPV) of both UKBDRS scores at various thresholds. We have also presented this data as a UKBDRS Calculator, an excel sheet where users can enter responses for the 9 measures of UKBDRS 
and

receive

a

14-year

risk

of

dementia

(https://github.com/MelisAnaturk/dementia_risk_score/blob/main/results/UKB-DRS_Calculator.xl sx).

The age-only and UKBDRS models were well-calibrated, i.e., they had an intercept and slope close to 0 and 1 , respectively, and a non-significant Spiegelhalter Z-test $(p>0.05)$. The CAIDE and DRS deviated from the line of perfect calibration, as suggested by an intercept and slope higher/lower than 0 and 1 and a significant Spiegelhalter Z-test (SI Table 9, SI Fig. 3). 
Table 2: Discrimination accuracy of models across the training and test sets. AUCs are reported with $95 \%$ confidence intervals indicated in square brackets. $0.9 \%$ of the UKB sample had missing data for one variable of the ANU-ADRI score (BMI). Therefore, all individuals with missing data on the ANU-ADRI were first excluded prior to evaluating the AUC for the ANU-ADRI. Abbreviations: Dementia Risk Score = DRS, Australian National University Alzheimer's Disease Risk Index = ANU-ADRI, Cardiovascular Risk Factors, Aging and Dementia $=$ CAIDE.

\begin{tabular}{|c|c|c|c|}
\hline & UKB Train & UKB Test & WHII \\
\hline $\mathbf{N}$ & $\mathbf{1 7 6 , 6 1 1}$ & $\mathbf{4 4 , 1 5 1}$ & $\mathbf{3 , 0 2 8}$ \\
\hline \multicolumn{4}{|c|}{ Baseline Model } \\
\hline Age only & $0.75[0.74,0.75]$ & $0.75[0.73,0.77]$ & $0.72[0.68,0.77]$ \\
\hline \multicolumn{4}{|c|}{ UKBDRS } \\
\hline UKBDRS & $0.77[0.76,0.78]$ & $0.77[0.75,0.78]$ & $0.75[0.7,0.79]$ \\
\hline UKBDRS-APOE & $0.80[0.79,0.81]$ & $0.80[0.79,0.82]$ & $0.80[0.75,0.84]$ \\
\hline \multicolumn{5}{|c|}{ Other risk scores } \\
\hline CAIDE & $0.58[0.57,0.59]$ & $0.59[0.57,0.69]$ & $0.66[0.61,0.71]$ \\
\hline DRS & $0.75[0.74,0.75]$ & $0.75[0.73,0.76]$ & $0.72[0.68,0.77]$ \\
\hline ANU-ADRI & $0.57[0.56,0.58]$ & $0.57[0.55,0.59]$ & $0.49[0.43,0.55]$ \\
\hline
\end{tabular}


Table 3: Pairwise comparisons in the UKB test set and WHII validation sample to survive FDR corrections. The AUC of the UKBDRS was compared to each external risk score and an age-only model. If a comparison is not shown, there was no statistical difference in performance.

\begin{tabular}{|c|c|c|c|c|c|c|}
\hline $\begin{array}{c}\text { Risk } \\
\text { score } 1\end{array}$ & $\begin{array}{l}\text { AUC for } \\
\text { Risk } \\
\text { score } 1\end{array}$ & $\begin{array}{c}\text { Risk score } \\
2\end{array}$ & $\begin{array}{l}\text { AUC for Risk } \\
\text { score } 2\end{array}$ & $\mathbf{Z}$ & $p$ & $\boldsymbol{p}_{\text {corr }}$ \\
\hline \multicolumn{7}{|l|}{ UKB } \\
\hline UKBDRS & 0.77 & age only & 0.75 & 4.83 & $1.34 \times 10-6$ & $1.34 \times 10-6$ \\
\hline UKBDRS & 0.77 & ANUADRI & 0.57 & 17.19 & $3.36 \times 10^{-65}$ & $1.34 \times 10^{-65}$ \\
\hline UKBDRS & 0.77 & CAIDE & 0.59 & 15.77 & $5.24 \times 10^{-56}$ & $1.05 \times 10^{-55}$ \\
\hline UKBDRS & 0.77 & DRS & 0.75 & 5.71 & $1.34 \times 10^{-6}$ & $1.34 \times 10^{-6}$ \\
\hline \multicolumn{7}{|l|}{ WHII } \\
\hline UKBDRS & 0.75 & age only & 0.72 & 2.64 & $8.2 \times 10^{-3}$ & $1.09 \times 10^{-2}$ \\
\hline UKBDRS & 0.75 & ANUADRI & 0.49 & 7.08 & $1.42 \times 10^{-12}$ & $5.7 \times 10^{-12}$ \\
\hline UKBDRS & 0.75 & CAIDE & 0.66 & 3.27 & $1.1 \times 10^{-3}$ & $2.19 \times 10^{-3}$ \\
\hline UKBDRS & 0.75 & DRS & 0.72 & 2.15 & $3.2 \times 10^{-2}$ & $3.19 \times 10^{-2}$ \\
\hline
\end{tabular}




\section{DISCUSSION}

We developed a risk score for predicting up to 14-year all-cause incident dementia in individuals 50-73 years old, and evaluated it against three published risk scores[9-11]. A model consisting of age, education, diabetes, depression, stroke, parental history of dementia, material deprivation, hypertensive status, and cholesterol status achieved a good-to-strong predictive accuracy and was well calibrated, with and without APOE4. Importantly, the discriminative performance of our score was consistently higher than three other established risk models, further supporting its utility in predicting individual-level risk of dementia in a mid-life UK population. We offer an excel sheet (https://github.com/MelisAnaturk/dementia_risk_score/blob/main/results/UKB-DRS_Calculator.xI sx) to calculate an individual's risk of dementia (we stress that this tool is for illustrative purposes only, not for clinical use, and does not replace clinical judgement)). Individuals can also be classified into low and high-risk groups, according to sensitivity and specificity thresholds reported in SI Table 8. As the information required for computing the UKBDRS can be easily collected at a population level, and some can be managed by targeted interventions, the UKBDRS may be a promising screening tool for stratifying middle-aged UK individuals for preventative interventions. Our study also raised important considerations about the reliability of dementia risk scores, as evidenced by the poor out-of-sample performance of some external scores. We therefore discuss recommendations for using dementia risk scores in clinical trials.

The UKBDRS includes established modifiable risk factors for dementia, namely stroke, type 2 diabetes, hypertension, and depression[24]. Our score also included material deprivation, fewer years of education and parental history as markers of increased risk, all of which have been repeatedly associated with dementia[2, 25-27].

In contrast to other risk scores, our model did not identify sex as a predictive factor for dementia[3, 9, 11]. To further explore this, we tested our model when manually including sex in 
the score, as well as by stratifying our score by sex. Neither approach improved performance and thus sex was left out of our final model. Interestingly, a recent study of the UKB found that risk of dementia was higher in men than women, and that a greater proportion of men had impaired cardiovascular health[14]. Thus, any UKB-specific sex effects may be accounted for by other risks in our model. Our model also did not identify other factors previously linked to dementia risk, such as BMI and physical inactivity[2, 3]. It is possible that even if these lifestyle factors have been linked to risk of dementia, (1) their predictive utility for dementia may change with age and time to diagnosis, and (2) their non-specific role in the development of other diseases in later life may lessen their sensitivity and specificity for dementia. It is important to note the goal of our study was to create an accurate, parsimonious model, as opposed to identifying all factors associated with dementia.

The UKBDRS consistently outperformed three scores previously developed for dementia risk prediction (i.e., DRS, ANU-ADRI, CAIDE) in both internal and external validation samples. Our AUCs for the external dementia scores fell within the range of values reported from previous validation attempts (CAIDE: 0.49-0.78[6, 7, 11]; ANU-ADRI: 0.49-0.78[6, 7, 10]; DRS: 0.56-0.84[6, 9]. Both ANU-ADRI and CAIDE also performed worse in comparison to a model based solely on age, a striking observation but in line with their recent evaluation in the Rotterdam cohort[6]. This could be for several reasons. First, certain predictors may not map precisely from one dataset to another. For example, CAIDE defines physically active individuals as those who exercise at least twice a week, lasting at least 20-30 minutes each time. UKB participants were instead asked how many minutes were spent being physically active on a typical day. Second, ANU-ADRI was developed for older individuals (60+), while our cohort is slightly younger (50+). However, our sensitivity analysis found that the ANU-ADRI also performed poorly when restricting our cohort to an age range matching its development sample. Third, CAIDE was designed to predict dementia over 20 years, while our cohorts had 
timeframes of only 14 and 17 years respectively. Fourth, there were differences in diagnosis procedures between cohorts; CAIDE performed diagnosis according to DSM designations. Similarly, ANU-ADRI was developed specifically for Alzheimer's Disease as opposed to the all-cause dementia outcome in our study. Finally, despite including all the predictors from the CAIDE and ANU-ADRI (except pesticide exposure) in our initial LASSO regression, only two of the seven predictors from CAIDE and four of the 12 predictors from ANU-ADRI were identified as important predictors in UKB. By contrast, 5 of the 14 predictors from the DRS model were initially selected as relevant predictors by our LASSO model. Thus, the performance of risk scores may be driven by these population-specific differences.

Our score included age, which may be questioned as it is not a modifiable target. However, if the goal is to stratify for risk, then the inclusion of age is obvious. In this context, we note the good performance of the baseline age-only model. Age may proxy relevant information from several age-associated medical predictors (e.g., cardiovascular health). A recently developed risk score which explicitly excluded age to focus on modifiable factors achieved an AUC of 0.59 , demonstrating that age can be expected to be the driving force in many risk scores and poor discrimination can be expected when not considering age[28]. We therefore suggest that risk scores with the explicit goal of risk stratification include age and other non-modifiable factors to achieve a stronger predictive performance.

It is possible our score could be improved by adding cognitive tests, brain MRI, and blood-based biomarkers[3,5]. A recent review noted that high-performing risk scores included these variables, but as they are expensive and/or time consuming they would have limited application in population-level settings[3]. Moreover, UKB uses cohort-specific cognitive tests rather than established cognitive batteries typically used for dementia screenings which would have made it difficult to validate a cognition-based UKB score in other cohorts that do not have similar cognitive measures. Therefore, our UKBDRS may best be used as an initial screening 
tool to stratify individuals into risk groups, and those identified as high risk could then benefit from more time-consuming follow-up assessments described above for more detailed characterisation.

Several limitations need to be considered when interpreting our findings. First, we note that the UK Biobank does not have a gold standard clinically adjudicated dementia diagnosis. Nonetheless, our approach, which used a combination of primary care, hospital inpatient records, death certificates, and self-report, has demonstrated a strong PPV of $82.5 \%$ when compared to clinical adjudication[29], and has been used extensively in this cohort[14, 15]. As the reported PPV of this approach is lower when stratifying outcomes by dementia sub-type, we restricted our outcome to all-cause dementia[29].

Second, the UKB and WHII cohorts differed in the availability of data used for dementia classification, which may partly explain the difference in the prevalence of all-cause dementia $(1.7 \%$ in UKB vs $3.2 \%$ in $\mathrm{WHII})$. While death, primary care and hospital records were supplemented by self-report in UKB, only hospital inpatient records and self-report were available for the WHII which may have affected how sensitive we were in identifying 'true positives'. We also note cohort differences in demographics, lifestyle, and health variables which may partly explain the lower AUC achieved in the external WHII sample.

Third, there were differences in the prevalence of factors used to compute the external scores, potentially affecting their predictive ability. For example, the UKB version of the ANU-ADRI excluded the cognitive component while the WHII version of this score missed information on TBI and pesticide exposure. Moreover, WHII participants consumed more units of alcohol per week relative to UKB (18.8 units/week vs 14.3 units/week) and had substantially fewer women ( $28 \%$ vs $51 \%)$. The UKB also does not offer information on whether participants need help with their finances or medications, which prevented us from computing other risk models. 
Fourth, it is possible the samples of UKB and WHII data assessed introduced a healthy volunteer bias. While there were statistically significant differences between the included and excluded participants in terms of age, sex, and education these were small (e.g., age: 59.97 vs. 60.71). The UKB and WHII cohorts are also healthier than the general population. For example, the prevalence of risk factors (e.g., BMI, smoking) and rates of disease in the UKB are lower than in the general population[30]. Both the UKB and WHII cohorts are predominantly Caucasian and are less likely to live in socioeconomically deprived areas than the general UK population[13, 30]. It is well known that dementia risk, onset and prevalence vary by race, ethnicity and socioeconomic status[7]. Therefore, while the consistent performance of UKBDRS across these two independent cohorts adds confidence to its robustness, we emphasise the need to evaluate it across more diverse cohorts both in and out of the UK before translating it for wider use.

\section{CLINICAL IMPLICATIONS}

We have developed and validated a novel dementia risk score, which outperformed other established risk scores in both an internal and external validation dataset. We recommend the UKBDRS for future studies interested in midlife dementia risk in a UK population. The UKBDRS includes age, education, material deprivation, history of diabetes, depression, stroke, parental history of dementia, hypertensive status, and cholesterol status, making it easy to assess in large populations. We also present a version of the UKBDRS which includes APOE4 and can offer improved performance if this information is available. With further validation, the UKBDRS may be useful as a dementia screening tool for a wide range of middle-aged adults in either a clinical or research setting.

Importantly, this study raises concerns about the overall generalizability of dementia risk models. Our findings and others have shown that dementia risk scores typically have reduced predictive accuracy when applied under different settings. This suggests that there is unlikely to 
be a single 'one-size-fits-all' dementia risk score for all populations. Instead, we suggest that any clinical trial should carefully consider the demographics of their participants and either identify a closely matched risk score, or pool together predictions from multiple risk models, with the UKBDRS potentially serving as one of these models. 
Acknowledgements: We would like to thank all participants of the UK Biobank and Whitehall II studies for dedicating their time towards these projects. For the Whitehall II study, we thank all the participating civil service departments; the British Occupational Health and Safety Agency; the British Council of Civil Service Unions; all participating civil servants in the Whitehall II Study; and all members of the Whitehall II Study team at University College London who so helpfully collaborated with us. The Whitehall II Study team comprises research scientists, statisticians, study coordinators, nurses, data managers, administrative assistants, and data entry staff, who make the study possible.

\section{Statements and Declarations}

\section{Authors' contributions}

M.A., R. P. and S.S. planned the statistical analyses and prepared the original draft of the manuscript. M.A. and R.P conducted the statistical analyses. G.G., D.N., A.T., A-M.G.dL. and K.P.E. provided guidance and discussion towards the analysis plan. M.K. and A.S-M. lead the WHII study, and all authors reviewed and provided feedback on all versions of the manuscript.

\section{Ethics Declarations}

\section{Ethics approval and consent to participate}

The UKB study received ethical approval from the North West Multi-Centre Research Ethics Committee (MREC) and the WHII study obtained ethical approval from the University College London Medical School Committee on the Ethics of Human Research. All participants provided their informed written consent.

\section{Competing Interests}

The authors declare no competing interests. 


\section{Funding}

The UK Biobank resource is supported by funding from the UK Medical Research Council and the Wellcome Trust. The Whitehall II study is funded by the US National Institutes on Aging (R01AG056477, RF1AG062553), the UK Medical Research Council (R024227, S011676, MR/K013351/1), Wellcome Trust (221854/Z/20/Z) and the British Heart Foundation (RG/13/2/30098), with the Whitehall II analyses conducted using the MRC Dementias Platform UK (DPUK). DPUK is a Public Private Partnership funded by the Medical Research Council (MR/L023784/1) and MR/009076/1) with further information on this resource available at www dementiasplatform.co.uk. The authors report the following funding: a UK Alzheimer's Society Research Fellowship (S.S.; Grant 441), Academy of Medical Sciences/the Wellcome Trust/the Government Department of Business, Energy and Industrial Strategy/the British Heart Foundation/Diabetes UK Springboard Award (S.S; Grant SBF006\1078), HDH Wills 1965 Charitable Trust (Nr: 1117747, K.P.E. \& M.A.), the Swiss National Science Foundation (A-M.G.dL.; PZ00P3_193658), UK Medical Research Council (M.K.; R024227, S011676; K.P.E.; G1001354; M.K., K.P.E. \& A.S.-M.; MR/K013351/1), the US National Institute on Aging (A.S-M. and M.K.; R01AG062553), UK Research and Innovation (J.H.C., M.A.; MR/R024790/1), Canadian Institutes of Health Research Fellowship (R.P., Grant number 458882), Fonds de Recherche du Quebec - Santé Doctoral Training Award (R.P., Grant Number 258239), and the European Commission (K.P.E.; Horizon 2020, Grant agreement number: 732592) and Wellcome Trust (A.T.; 216462/Z/19/Z; M.K.; 221854/Z/20/Z). This work was supported by the NIHR Oxford Health Biomedical Research Centre and the Wellcome Centre for Integrative Neuroimaging (WIN). The WIN is supported by core funding from the Wellcome Trust (203139/Z/16/Z). The funders of this study had no role in the study design or collection, interpretation, analysis or reporting of the data 


\section{References}

1. Nichols E, Steinmetz JD, Vollset SE, et al (2022) Estimation of the global prevalence of dementia in 2019 and forecasted prevalence in 2050: an analysis for the Global Burden of Disease Study 2019. The Lancet Public Health 7:e105-e125

2. Livingston G, Huntley J, Sommerlad A, et al (2020) Dementia prevention, intervention, and care: 2020 report of the Lancet Commission. Lancet

3. Hou X-H, Feng L, Zhang C, Cao X-P, Tan L, Yu J-T (2019) Models for predicting risk of dementia: a systematic review. J Neurol Neurosurg Psychiatry 90:373-379

4. Tang EYH, Harrison SL, Errington L, et al (2015) Current Developments in Dementia Risk Prediction Modelling: An Updated Systematic Review. PLoS One 10:e0136181

5. Anstey KJ, Zheng L, Peters R, Kootar S, Barbera M, Stephen R, Dua T, Chowdhary N, Solomon A, Kivipelto M (2021) Dementia Risk Scores and Their Role in the Implementation of Risk Reduction Guidelines. Front Neurol 12:765454

6. Licher S, Yilmaz P, Leening MJG, Wolters FJ, Vernooij MW, Stephan BCM, Ikram MK, Ikram MA (2018) External validation of four dementia prediction models for use in the general community-dwelling population: a comparative analysis from the Rotterdam Study. Eur J Epidemiol 33:645-655

7. Stephan BCM, Pakpahan E, Siervo M, et al (2020) Prediction of dementia risk in low-income and middle-income countries (the 10/66 Study): an independent external validation of existing models. The Lancet Global Health 8:e524-e535

8. Vonk JMJ, Greving JP, Gudnason V, Launer LJ, Geerlings MI (2021) Dementia risk in the general population: large-scale external validation of prediction models in the AGES-Reykjavik study. Eur J Epidemiol 36:1025-1041

9. Walters K, Hardoon S, Petersen I, Iliffe S, Omar RZ, Nazareth I, Rait G (2016) Predicting dementia risk in primary care: development and validation of the Dementia Risk Score using routinely collected data. BMC Med 14:6

10. Anstey KJ, Cherbuin N, Herath PM, Qiu C, Kuller LH, Lopez OL, Wilson RS, Fratiglioni L (2014) A self-report risk index to predict occurrence of dementia in three independent cohorts of older adults: the ANU-ADRI. PLoS One 9:e86141

11. Kivipelto M, Ngandu T, Laatikainen T, Winblad B, Soininen H, Tuomilehto J (2006) Risk score for the prediction of dementia risk in 20 years among middle aged people: a longitudinal, population-based study. Lancet Neurol 5:735-741

12. Sudlow C, Gallacher J, Allen N, et al (2015) UK biobank: an open access resource for identifying the causes of a wide range of complex diseases of middle and old age. PLoS Med 12:e1001779

13. Marmot M, Brunner E (2005) Cohort Profile: the Whitehall II study. Int J Epidemiol $34: 251-256$ 
14. Gong J, Harris K, Peters SAE, Woodward M (2021) Sex differences in the association between major cardiovascular risk factors in midlife and dementia: a cohort study using data from the UK Biobank. BMC Med 19:110

15. Lourida I, Hannon E, Littlejohns TJ, Langa KM, Hyppönen E, Kuzma E, Llewellyn DJ (2019) Association of Lifestyle and Genetic Risk With Incidence of Dementia. JAMA 322:430-437

16. Sabia S, Fayosse A, Dumurgier J, van Hees VT, Paquet C, Sommerlad A, Kivimäki M, Dugravot A, Singh-Manoux A (2021) Association of sleep duration in middle and old age with incidence of dementia. Nat Commun 12:2289

17. Sabia S, Dugravot A, Dartigues J-F, Abell J, Elbaz A, Kivimäki M, Singh-Manoux A (2017) Physical activity, cognitive decline, and risk of dementia: 28 year follow-up of Whitehall II cohort study. BMJ 357:j2709

18. Tibshirani R (1996) Regression shrinkage and selection via the lasso. J R Stat Soc 58:267-288

19. DeLong ER, DeLong DM, Clarke-Pearson DL (1988) Comparing the areas under two or more correlated receiver operating characteristic curves: a nonparametric approach. Biometrics 837-845

20. Kuhn M (2008) Building Predictive Models in R Using the caret Package. J Stat Softw 28:1-26

21. Friedman J, Hastie T, Tibshirani R (2010) Regularization Paths for Generalized Linear Models via Coordinate Descent. J Stat Softw 33:1-22

22. Robin X, Turck N, Hainard A, Tiberti N, Lisacek F, Sanchez J-C, Müller M (2011) pROC: an open-source package for $\mathrm{R}$ and $\mathrm{S}+$ to analyze and compare ROC curves. BMC Bioinformatics 12:77

23. Harrell FE Jr (2016) rms: Regression modeling strategies. R package version

24. Poblador-Plou B, Calderón-Larrañaga A, Marta-Moreno J, Hancco-Saavedra J, Sicras-Mainar A, Soljak M, Prados-Torres A (2014) Comorbidity of dementia: a cross-sectional study of primary care older patients. BMC Psychiatry 14:84

25. Norton S, Matthews FE, Barnes DE, Yaffe K, Brayne C (2014) Potential for primary prevention of Alzheimer's disease: an analysis of population-based data. Lancet Neurol 13:788-794

26. Vrijsen J, Abu-Hanna A, de Rooij SE, Smidt N (2021) Association between dementia parental family history and mid-life modifiable risk factors for dementia: a cross-sectional study using propensity score matching within the Lifelines cohort. BMJ Open 11:e049918

27. Pase MP, Rowsthorn E, Cavuoto MG, Lavale A, Yassi N, Maruff P, Buckley RF, Lim YY (2022) Association of Neighborhood-Level Socioeconomic Measures With Cognition and Dementia Risk in Australian Adults. JAMA Netw Open 5:e224071

28. Vos SJB, van Boxtel MPJ, Schiepers OJG, et al (2017) Modifiable Risk Factors for Prevention of Dementia in Midlife, Late Life and the Oldest-Old: Validation of the LIBRA Index. J Alzheimers Dis 58:537-547 
29. Wilkinson T, Schnier C, Bush K, et al (2019) Identifying dementia outcomes in UK Biobank: a validation study of primary care, hospital admissions and mortality data. Eur J Epidemiol 34:557-565

30. Fry A, Littlejohns TJ, Sudlow C, Doherty N, Adamska L, Sprosen T, Collins R, Allen NE (2017) Comparison of sociodemographic and health-related characteristics of UK Biobank participants with those of the general population. Am J Epidemiol 186:1026-1034 
Figure 1 Receiver-operating characteristic curves (ROC) for each risk score in the UKB test set. Sensitivity and specificity (plotted from 1 to 0 ) at varying thresholds to build and ROC curve for each risk score computed in the UKB Test set. The highest performing score, as indicated by the greatest area under the curve, was the UKBDRS-APOE model, followed by the UKBDRS, age only, DRS, CAIDE, and ANU_ADRI. The UKBDRS-APOE performance is assessed in the subset of individuals with genotype information available

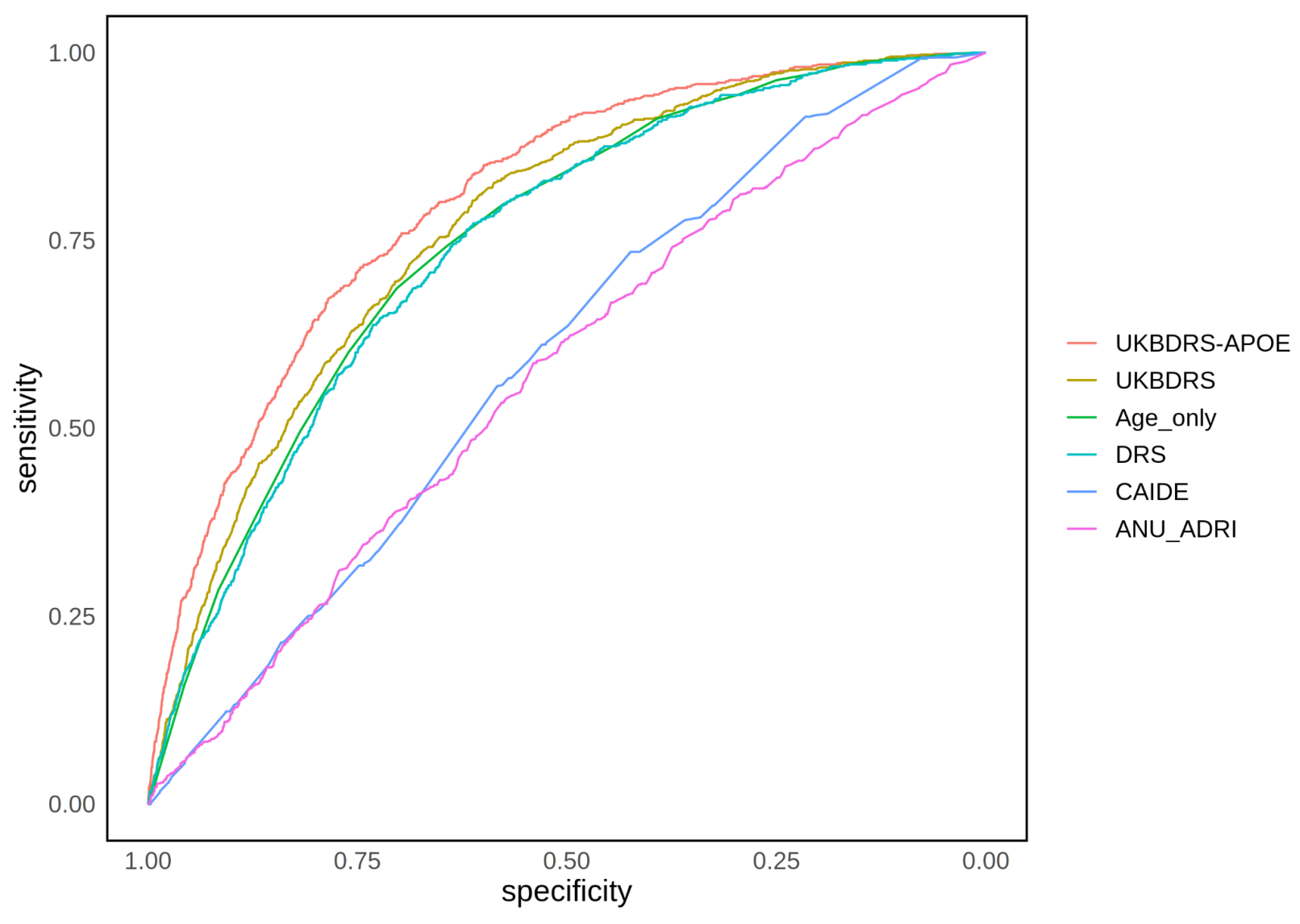

